\title{
Abstracts of presentations*
}

\section{Working party 1 session: health and nutrients}

\section{Epidemiological case for a food and health policy in the European Union}

Eric Brunner*

Department of Epidemiology and Public Health, University College London, 1-19 Torrington place, WCIE 6BT, UK

* Correspondence: Email eric @ public-health.ucl.ac.uk

Keywords

diet, nutrition, public health, primary prevention

During the last 150 years life expectancy in Europe increased dramatically. The improvement was the result of public health measures such as effective sanitation and food hygiene rather than developments in medical care. The WHO MONICA Study finds that less than a third of the recent decline in coronary heart disease can be explained by therapeutic advances. However, health remains distributed unequally between and within
European populations, and in some countries life expectancy is falling. Dietary differences are key explanations of social and geographical inequalities. The evidence points to diet as a means to reduce the burden of disease in EU member states, recognising that food culture and patterns of diet-related disease vary between them.

\section{The role of nutrition in the prevention of cancer}

\section{E. Riboli*}

Unit of Nutrition and Cancer, International Agency for Research on Cancer, 150 Cours Albert-Thomas, 69372 Lyon, France

* Correspondence: Email riboli@iarc.fr

Keywords

nutrition, prevention, cancer, EPIC

Research on nutrition and cancer has developed substantially over the past 20 years, initially stimulated by a number of epidemiological studies which drew attention to the large world-wide variations in cancer incidence, suggesting that these variations could be related to differences in diet and lifestyle between populations. Hundreds of epidemiological studies have been conducted so far on the relation between diet and the risk of developing different types of cancer, particularly cancers of the digestive and respiratory tracts, the breast, the female reproductive organs and the prostate. The most consistent results so far are that:

A diet rich in vegetables and fruit is associated with lower cancer risk. The epidemiological evidence is particularly consistent for a protective effect of vegetables on cancers of the colon, and of vegetables and fruit on cancers of the mouth, pharynx, larynx, lung and stomach.

Elevated consumption of meat is associated with a modest increase in colorectal cancer risk.

Elevated consumption of salt and salt-preserved foods increases stomach cancer risk.

Obesity definitely increases the risk of cancers of the endometrium at any age and of the breast after menopause.

A sedentary lifestyle increases the risk of colorectal cancer.

The biological background behind these epidemiological results is certainly complex. Regarding fruit and vegetables, various hypotheses have been put forward to explain why their consumption is associated with a reduced risk of cancer; among others, these include the biological effects of natural antioxidants, various 
vitamins and minerals, dietary fibre, resistant starches and various natural components such as coumarins, flavonoids, isoflavones, isothiacyanates, lignans and phytosterols. During the past 15 years, in parallel to laboratory research aimed at identifying potential anticarcinogens, several large randomized trials were initiated to test whether high pharmacological doses of various combinations of vitamins, minerals and dietary fibre could reduce cancer risk or recurrence of preneoplastic lesions.

The results of several randomized studies have been published recently, and they have so far been quite disappointing, indicating that supplementation either had a very modest effect, or did not reduce cancer risk, or even increased cancer risk in the case of two beta-carotene trials.

The associations found between obesity, sedentary lifestyle and increased cancer risk may be mediated via endogenous hormones. It has been suggested that insulin resistance leading to higher insulin secretion may be one of the key factors to explain this association. Very recent prospective cohort studies in which IGFs, steroid hormones and their binding protein have been measured have lent support to this hypothesis. These results suggest that the biological mechanisms behind the diet and cancer relationship are much more complex than the "one molecule, one effect" biological model underlying traditional chemical carcinogenesis models. A strategy combining observational epidemiology, laboratory research and randomized intervention studies on diet and lifestyle is likely to be a more appropriate means of tackling such a complex issue.

Eight years ago the International Agency for Research on Cancer initiated a large European project with the aim of clarifying the role of diet in the etiology of cancer. The project, named EPIC (European Prospective Investigation into Cancer and Nutrition), is a multi-centre prospective cohort study designed to investigate the relation between diet, nutritional and metabolic characteristics, various lifestyle factors and the risk of cancer. The study is based in 22 collaborating centres in nine European countries and includes populations characterized by large variations in dietary habits and cancer risk.

Detailed data on diet, lifestyle and anthropometry have been collected from all study subjects and stored for further statistical analyses. The study was initiated in 1992, and by 1999 about 480,000 subjects had been included with questionnaire data. From 400,000 of them blood samples had been collected and stored in large liquid nitrogen containers at $-196^{\circ} \mathrm{C}$ to ensure maximum stability. Plasma, serum, buffy coat and red blood cells are stored in small plastic straws ( 28 per subject) with a capacity of only $0.5 \mathrm{ml}$ in order to have available for the future small aliquots more adapted to new laboratory "micromethods". The main advantage of this approach is that it leaves the door open for as many different types of laboratory analyses as may be scientifically justified and technically possible in the next 10-15 years.

It is expected that about 17,000 cases of cancer will occur in the cohort by the year 2002. Followup is done through cancer registries and national mortality registries. Studies are being planned on the role of prediagnostic levels of steroid hormones, insulin-like growth factors and their binding proteins in the etiology of cancers of the breast, prostate and colorectum. These studies on metabolic factors will be paralleled by genetic investigations into susceptibility to cancer focusing initially on polymorphisms of genes involved in known metabolic functions (metabolism of hormones, some vitamins, exogenous carcinogens, etc.) which may possibly play a role in carcinogenesis. 


\section{Nutritional science: emerging issues}

\section{John H Cummings*}

Ninewells Hospital and Medical School, Dundee

* Correspondence: Email j.h.cummings@ dundee.ac.uk

Diet is important because it determines growth, resistance to infection, timing of the onset of degenerative diseases, recovery from injury and has a social and psychological dimension. It is a key component of public health policy and has economic impact through agriculture and the food industry.

At a time when advances in biological sciences are more rapid than ever, nutrition is ideally placed to integrate new post-genomic knowledge into tissue and organ function and, finally, whole body metabolism and a public health policy. Current scientific progress has raised many new issues in nutrition such as the use of genetically modified plants and bacteria, the creation of novel and designer foods, the functional food concept, as well as new problems with microbiological and toxicological safety including prion diseases, and the global issues of diminishing energy requirements of individuals in the face of increasing obesity. Food intolerance is growing in importance whilst the expanding market for organic food reflects increasing concern by consumers of the safety of food.

Despite the key role of diet as a modifiable environmental factor in disease prevention, the complexity in interelationships of the diet and disease story are increasing. Consider the example of colorectal cancer and diet. ${ }^{1}$ It is only a little over ten years since the first genetic abnormality associated with a predisposition to colorectal cancer was described. Today more than a dozen genetic abnormalities have been identified in this disease and we still have only partial information on the role of these genes in cellular function. Equally, the dietary factors which are emerging as key in risk for colorectal cancer include red meat and alcohol which probably increase risk along

$$
\text { diet, genetics, environment, cancer, diarrhoea }
$$

with fat, sugar and obesity which may also contribute whilst vegetables decrease risk along possibly with dietary fibre, starch and carotenoids. Equally important is the emergence of lifestyle and environmental factors in disease risk with physical activity decreasing risk of colorectal cancer whilst infection with Helicobacter pylori now emerging as a risk factor for stomach cancer. The diet and cancer story, therefore, emerges as being polygenetic, multinutrient, and involving both lifestyle and environmental factors.

Diet, however, may be much more pervasive in preventing disease than we have hitherto recognised. Even in conditions such as acute diarrhoea, which is one of the major causes of mortality in children all

over the world, diet not only may provide the infectious agent, but may also contribute to colonisation resistance in the gut through the influence of prebiotic carbohydrates on the growth of genera such as bifidobacteria and lactobacilli. ${ }^{2}$

Diet remains an important fact in disease prevention, but it might be remembered that other factors are also important and in any preventive health campaign, a co-ordinated approach is required.

\section{References}

1. Cummings JH, Bingham SA. Clinical Review: Diet and the Prevention of Cancer. British Medical Journal 1998; 317: 1634-1640.

2. Macfarlane GT, Cummings JH. Probiotics and Prebiotics: Can Regulating the Activities of Intestinal Bacteria Benefit Health? British Medical Journal 1999; 318: 999-1003. 